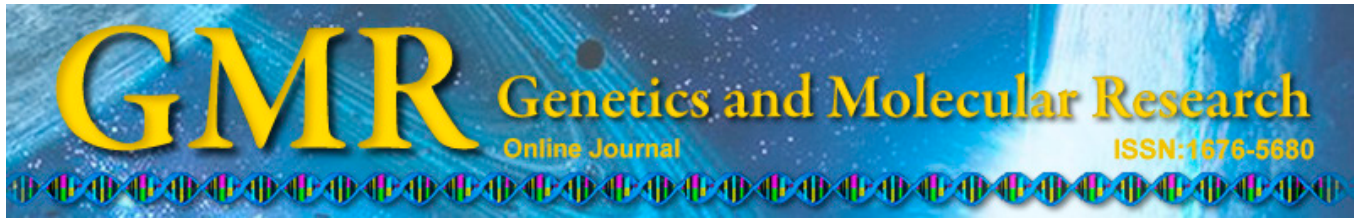

\title{
Variations in $W N T 3$ gene are associated with incidence of non-syndromic cleft lip with or without cleft palate in a northeast Chinese population
}

\author{
Y.P. Lu' ${ }^{1}$ W.T. Han ${ }^{1}$, Q. Liü ${ }^{2}$, J.X. Li ${ }^{1}$, Z.J. Li ${ }^{2}$, M. Jiang' ${ }^{1}$ and W. Xu \\ ${ }^{1}$ Key Laboratory of Reproductive Health, \\ Liaoning Province Research Institute of Family Planning, \\ Key Laboratory of Reproductive Health and Medical Genetics, \\ National Health and Family Planning Commission, Shenyang, China \\ ${ }^{2}$ Department of Oral Maxillofacial Surgery and Plastic Surgery, \\ School of Stomatology, China Medical University, Shenyang, China \\ Corresponding authors: W.T. Han / Q. Liu \\ E-mail: hwtzzf@sohu.com / liu66k@126.com
}

Genet. Mol. Res. 14 (4): 12646-12653 (2015)

Received May 9, 2015

Accepted August 18, 2015

Published October 19, 2015

DOI http://dx.doi.org/10.4238/2015.October.19.8

\begin{abstract}
Non-syndromic cleft lip with or without cleft palate (NSCL/P) is a common birth defect. Several WNT genes are involved in craniofacial embryogenesis, and therefore may play an important role in the etiology of NSCL/P. Two SNPs (rs3809857 and rs9890413) in the WNT3 gene were subjected to case-control and case-parent analysis by polymerase chain reaction-restriction fragment length polymorphism (PCR-RFLP) in 236 unrelated patients with NSCL/P, including 128 elementary families (185 mothers and 154 fathers), and 400 control individuals from northeast China. The rs3809857 SNP, under the assumption of a dominant model, was found to induce a 2-fold lower risk of NSCL/P OR GG vs GT $+\mathrm{TT}=0.605,95 \% \mathrm{CI}=0.436-0.839, \mathrm{P}=$ $0.003)$. Moreover, the family-based association test revealed an under-
\end{abstract}


transmission for the minor allele T. On the other hand, we observed a significant association in the case-control and case-parent analysis of the SNP rs9890413. In addition, the P values for the haplotype of rs3809857-rs9890413 were observed to be statistically significant $(\mathrm{P}=$ 0.004). In conclusion, our study confirmed the association between the WNT3 variant and NSCL/P in the population tested.

Key words: Cleft lip; Cleft palate; Family-based association test; WNT3

\section{INTRODUCTION}

Non-syndromic orofacial clefts (NSOCs) are common birth defects, affecting 1.25 in every 1000 newborns worldwide (Mossey and Modell, 2012). NSOCs are conventionally categorized into three groups: the cleft lip (CL), cleft lip with cleft palate (CLP), and cleft palate only (CPO) (Mossey et al., 2009). The first two, believed to be etiologically distinct from the third type (CPO), are collectively referred to as cleft lip with or without cleft palate (CL/P) (Pan et al., 2011). Environmental variations and genetic influences play important roles in non-syndromic cleft lip with ou without cleft palate (NSCL/P). Many candidate genes and loci have also been associated with facial clefts, through various genetic approaches. Over 40 genes, including IRF6, VAX1, MSX1, FOXE1, MYH9, MAFB, ABCA4, the 17q22 locus, BMP4, FGFR2, TGFA, TGFB3, MTHFR, GSTT1, PDGFC, FGF8, PVRL1, SUMO1, CRISPLD2, as well as the $8 \mathrm{q} 24$ locus, have been suggested to be associated with NSCL/P (Dixon et al., 2011).

There are four published genome-wide association (GWA) studies, three using a casecontrol design (Birnbaum et al., 2009; Grant et al., 2009; Mangold et al., 2010) and one using a case-parent trio design (Beaty et al., 2010), for NSCL/P. These studies confirmed the impact of IRF6, discovered a new region on chromosome 8q24, and identified two new loci (MAFB and ABCA4) associated with NSCL/P. Although not implicated by GWA studies, variants within the $W N T$ genes have been reported to be associated with NSCL/P, and the haplotype of the $W N T$ genes underlie the etiology of NSCL/P (Mostowska et al., 2012). The WNT gene family plays an important role in murine craniofacial embryogenesis (Brugmann et al., 2007); canonical $W N T$ signaling is activated during midfacial morphogenesis in mice (Lan et al., 2006). Chiquet et al. (2008) investigated seven $W N T$ genes (WNT3, WNT3A, WNT5A, WNT7A, $W N T 8 A, W N T 9 B$, and $W N T 11)$, and suggested that the single nucleotide polymorphisms (SNPs) in WNT3A, WNT5A, and WNT11 are significantly associated with NSCL/P. Another study tested fourteen SNPs in six WNT genes, and discovered the rs3809857 SNP in WNT3 to be highly associated with NSCL/P. Menezes et al. (2010) suggested that the variant alleles in WNT3 increased the risk of NSCL/P in a Caucasian population. The SNP rs142167 showed a highly significant association, while the SNP rs9890413 was also shown to be associated with NSCL/P. Beaty et al. (2006) also showed the WNT6-WNT10A gene cluster to be associated with incidence of cleft lip and palate.

Previous studies have shown that $W N T$ genes may contribute to the development of NSCL/P. Yao et al. (2011) investigated variants of the WNT3A gene, and reported two SNPs 
in $W N T 3 A$ (rs752107 and rs3121310) to be significantly associated with NSCL/P in a Chinese population. Feng et al. (2014) reported a C392T polymorphism in the WNT10A gene in people with NSCL/P in a northeast Chinese population. However, very few studies have evaluated the association between the WNT3 gene and NSCL/P in a Chinese population. The purpose of this study was to investigate the possibility of the rs3809857 and rs9890413 SNPs in the WNT3 gene being risk factors for NSCL/P in a northeastern Chinese (Table 1).

Table 1. Candidate single nucleotide polymorphism (SNP) function and minor allele frequency (MAF) in
northeast China.
\begin{tabular}{llllll}
\hline Gene & Chromosome & SNPs & SNP function & Allele & MAF \\
\hline WNT3 & $17 \mathrm{q} 21.31$ & rs 3809857 & Intronic & $\mathrm{G} / \mathrm{t}$ & 0.30 \\
$W N T 3$ & $17 \mathrm{q} 21.31$ & rs 9890413 & Upstream & $\mathrm{A} / \mathrm{g}$ & 0.03 \\
\hline
\end{tabular}

\section{MATERIAL AND METHODS}

\section{Patients and families}

As previously described by Lu et al. (2013), 236 unrelated patients with NSCL/P, including 128 elementary families (185 mothers and 154 fathers), and 400 control individuals were recruited from the Department of Oral and Maxillofacial Surgery of the Affiliated Stomatological Hospital of China Medical University, northeast China, between 2008 and 2012. All patients were subjected to a pre-operative examination and questionnaire; photographs were taken to record the shape of the lips, alveolar ridge, and hard and soft palates, in order to help with a diagnosis of cleft lip and palate. Syndromic patients, and controls with a family history of clefts and other anomalies were excluded. This study was approved by the Ethics Committee of the Liaoning Province Research Institute of Family Planning. Signed informed consent forms were obtained from all participants. DNA was obtained from the blood samples using a TIANamp Blood DNA kit (TianGen, Beijing, China).

\section{Genotyping}

The polymerase chain reaction-restriction fragment length polymorphism (PCRRFLP) assay was designed to detect the SNPs rs3809857 and rs9890413. Specific primer pairs were designed using the Primer 5.0 software. The primer sequences are listed below: rs3809857: forward: 5'-CCTTTTCTCGGTCTCAGTCG-3'; reverse: 5'-GCTTCCTTCTTGC AGCACTC-3'; product size 535 bp; rs9890413: forward: 5'-CCACCTTGTAGGGAGCTGT C-3'; reverse: 5'-ACAGGACAGGGCTAGGGAGT-3'; product size 307 bp. Subsequently, the PCR products were subjected to restriction digestion, using the Bam HI (rs3809857: cuts at the $384 \mathrm{bp}$ position in the presence of a T-allele) and MspI (rs9890413: cuts at the $206 \mathrm{bp}$ position in the presence of a G-allele) enzymes (Figures 1 and 2). Approximately 10\% of the randomly selected samples were genotyped by DNA sequencing for quality control; the obtained genotypes were consistent with the original results. 


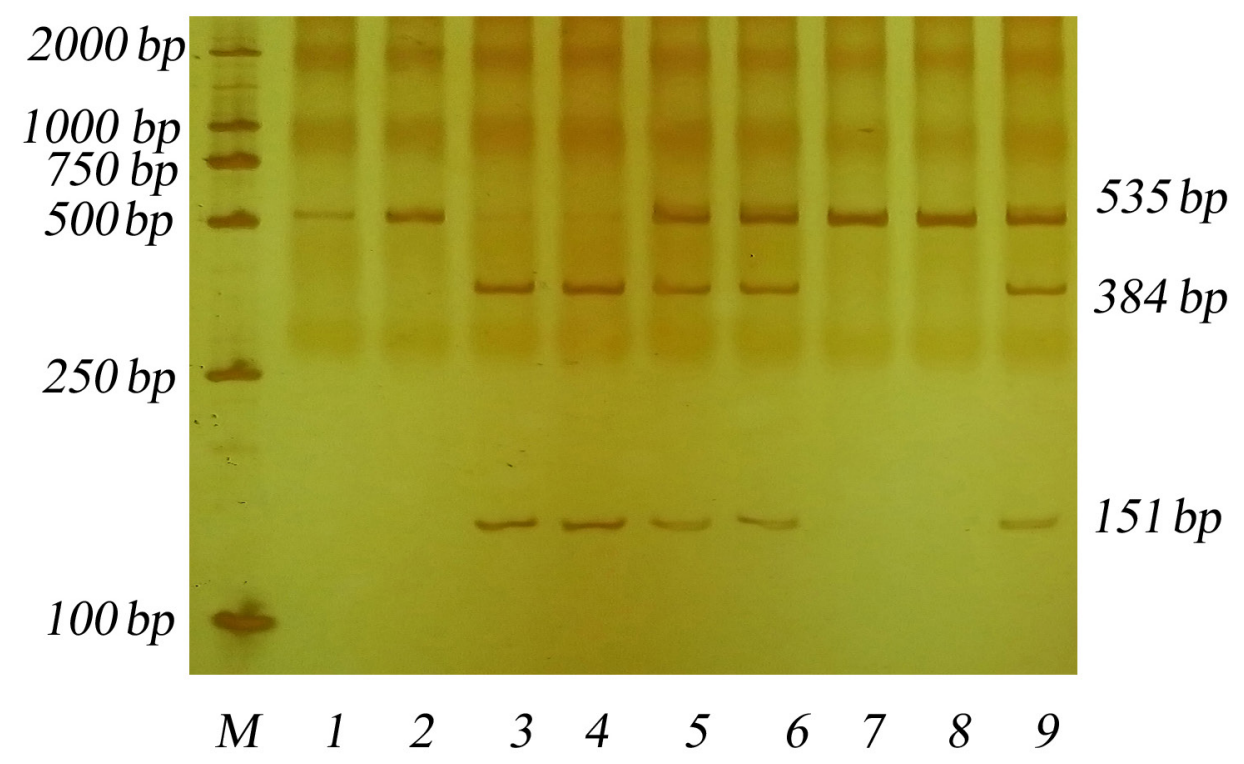

Figure 1. Patterns of restriction fragment length polymorphisms for the SNP rs3809857. Lane $M=$ DNA marker (DL2000). Lanes 1, 2, 7, and $8=$ subjects displaying the wild-type homozygous genotype GG; lanes 3 and $4=$ individuals expressing the mutated homozygous genotype TT [displaying two bands (384 and $151 \mathrm{bp}$ )]; lanes 5, 6, and $9=$ individuals expressing the heterozygous genotype GT (three bands: 535, 384, and $151 \mathrm{bp}$ ).

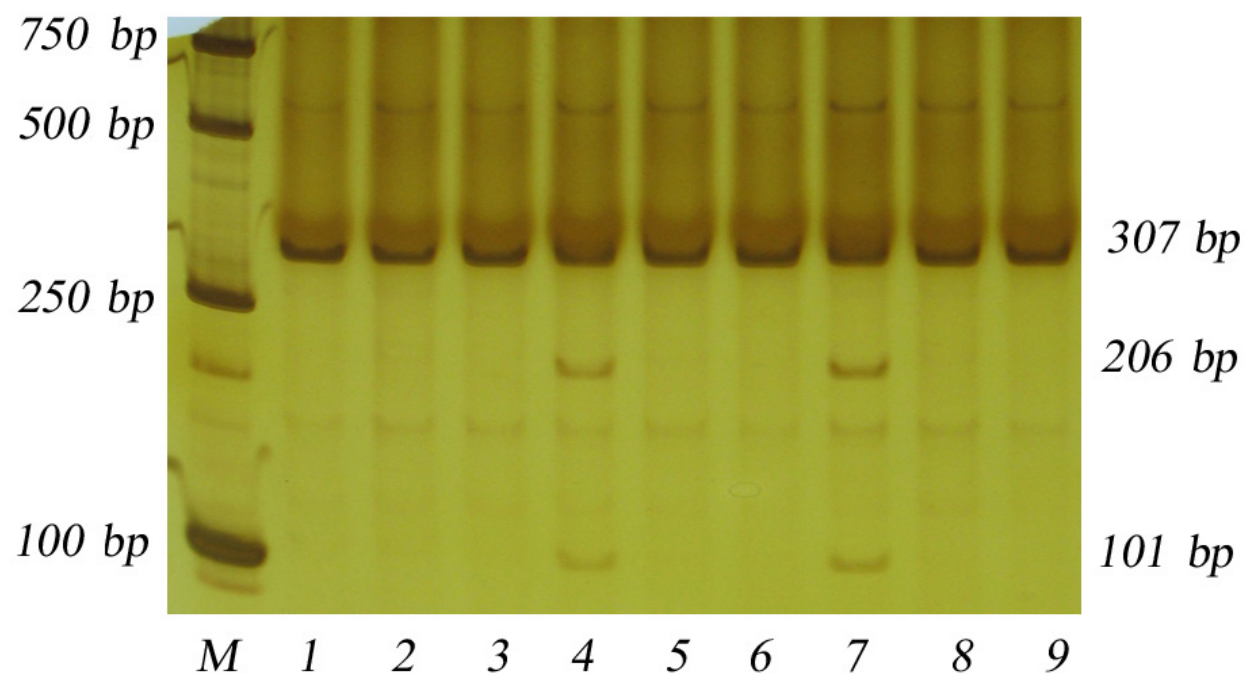

Figure 2. Patterns of restriction fragment length polymorphisms for the SNP rs9890413. Lane $M=$ DNA marker (DL2000). Lanes 1, 2, 3, 5, 6, 8, and 9 = individuals expressing the wild-type homozygous genotype AA; lanes 4 and $7=$ subjects displaying the heterozygous genotype AG. 


\section{Statistical methods}

Case-control statistical analyses, including the genotyping frequency and HardyWeinberg equilibrium, were calculated using the SPSS 17.0 statistical software package (http:// www-03.ibm.com/software/products/us/en/spss-stats-standard/). $\mathrm{P}<0.05$ was considered to be statistically significant. The transmission of the two SNPs in case-parent trios was calculated using the family-based association test, provided in the FBAT package (http://www.biostat. harvard.edu/fbat/default.htm). Haplotype analysis was performed using the HBAT application in the FBAT package.

\section{RESULTS}

The genotypes of the two SNPs in patients and controls were in accordance with the Hardy-Weinberg equilibrium (data not shown). A statistically significant difference was observed between the cases and controls for the presence of rs3809857 and rs9890413 (Table 2).

Table 2. Genotype distribution of the two single nucleotide polymorphisms (SNPs) in the WNT3 gene in patients
and controls.
\begin{tabular}{lllcccccc}
\hline Gene & rs No. & Genotype & Cases (frequency) & Controls (frequency) & Odds ratio (95\%Cl) & $\chi^{2}$ & $\mathrm{P}^{\mathrm{a}}$ & $\mathrm{P}^{\mathrm{b}}$ \\
\hline WNT3 & rs 3809857 & GG & $141(0.60)$ & $185(0.464)$ & Reference & - & - & \\
& & GT & $79(0.33)$ & $189(0.474)$ & $0.548(0.390-0.772)$ & 11.97 & $\mathbf{0 . 0 0 1}$ & $\mathbf{0 . 0 0 1}$ \\
& & TT & $16(0.07)$ & $26(0.062)$ & $0.807(0.417-1.562)$ & 0.404 & 0.525 & 0.638 \\
& & GT+TT & $95(0.40)$ & $209(0.536)$ & $0.605(0.436-0.839)$ & 9.11 & $\mathbf{0 . 0 0 3}$ & $\mathbf{0 . 0 0 3}$ \\
WNT3 & rs9890413 & AA & $205(0.869)$ & $376(0.94)$ & Reference & - & - & \\
& & AG & $27(0.114)$ & $23(0.0575)$ & $2.153(1.204-3.852)$ & 6.937 & $\mathbf{0 . 0 0 8}$ & $\mathbf{0 . 0 1 3}$ \\
& & GG & $4(0.017)$ & $1(0.0025)$ & $7.337(0.815-66.074)$ & 4.32 & $\mathbf{0 . 0 3 8}$ & 0.107 \\
& & AG+GG & $31(0.131)$ & $24(0.06)$ & $2.369(1.354-4.145)$ & 9.566 & $\mathbf{0 . 0 0 2}$ & $\mathbf{0 . 0 0 3}$ \\
\hline
\end{tabular}

${ }^{\mathrm{a} C h i}$-square analysis, ${ }^{\mathrm{b}}$ corrected $\mathrm{P}$ values. Bold values represent significance at $\mathrm{P}<0.05$.

The GG genotype of the WNT3 SNP rs3809857 was identified in 141 individuals in the NSCL/P group and 185 individuals from the control group, while the GT genotype was observed in 79 individuals of the NSCL/P group and 189 individuals of the control group; on the other hand, the TT genotype was discovered in 16 and 26 individuals of the NSCL/P and control groups, respectively. The odds ratios (OR) obtained for cases with the GT+TT and GT genotypes, compared to the GG homozygote were 0.605 (95\% confidence interval $(\mathrm{CI})=0.436$ $0.839, \mathrm{P}=0.003)$ and $0.548(95 \% \mathrm{CI}=0.390-0.772, \mathrm{P}=0.001)$, respectively. However, the OR of the TT genotype compared to the GG genotype was not statistically significant $(\mathrm{P}=0.638)$.

The AA homozygote of the WNT3 SNP rs9890413 was identified in 205 and 376 individuals of the NSCL/P and control groups, respectively, while the AG heterozygote was observed in 27 and 23 subjects belonging to the NSCL/P and control groups, respectively. The GG homozygote was detected in four patients affected by NSCL/P and one healthy control. The ORs of the AG, GG, and AG+GG genotypes were all statistically significant.

FBAT revealed strong associations between the two WNT3 SNPs and NSCL/P (Table 3). The rs3809857 SNP displayed an under-transmission of the minor frequency $T$ alleles and over-transmission of the common G allele. The rs9890413 SNP showed an over-transmission of the $\mathrm{G}$ allele from parent to child. The haplotype of the two SNPs were also determined. The G-G (order: rs3809857-rs9890413) haplotype was significantly correlated with NSCL/P (Table 4). 
Table 3. Results of the family-based association test (FBAT) of the two single nucleotide polymorphisms (SNPs) in the $W N T 3$ gene in case-parent trios.

\begin{tabular}{llccccc}
\hline Gene & SNPs & Allele & Frequency & Fam\# & Z & \multicolumn{1}{c}{ P } \\
\hline WNT3 & rs3809857 & G & 0.597 & 79 & 2.183 & 0.029 \\
& & T & 0.403 & 79 & -2.183 & 0.029 \\
WNT3 & rs9890413 & A & 0.931 & 18 & -3.838 & 0.00012 \\
& & G & 0.069 & 18 & 3.838 & 0.00012 \\
\hline
\end{tabular}

Frequency: gene frequency; Fam\#: number of families; Z: vector of the FBAT statistic.

Table 4. Haplotype results for the two single nucleotide polymorphisms (SNPs) in the WNT3 gene.

\begin{tabular}{lccccc}
\hline Gene & Haplotype & Frequency & Fam\# & Z & P \\
\hline WNT3 & G-A & 0.546 & 80 & 1.011 & 0.312 \\
& T-A & 0.385 & 76 & -2.778 & 0.005 \\
& G-G & 0.051 & 15 & 2.883 & 0.004 \\
\hline
\end{tabular}

Order of SNPs: rs3809857-rs9890413; Frequency: haplotype frequency; Fam\#: number of families; Z: vector of the FBAT statistic.

\section{DISCUSSION}

The $W N T$ gene family plays a critical role in the development of the lip; in addition, the role played by $W N T$ in the development of the ectoderm has attracted widespread attention. The family of conserved secreted glycoproteins constituting various $W N T$ signaling molecules plays a fundamental role in developmental and cell-biological processes (MacDonald et al., 2009). Liu and Millar (2010) reported that the dynamic activation of the $W N T /$ beta-catenin signaling pathway was correlated with the occurrence of a cleft lip and cleft palate. Song et al. (2009) reported that cleft lip with cleft palate may be induced by genetic inactivation of Lrp6, a co-receptor of the $W N T /$ beta-catenin signaling pathway. A previous case-control study associated mutations in $A X I N 2$, a gene belonging to the $W N T$ signaling pathway, with the occurrence of cleft lip/palate (Letra et al., 2009).

The members of the $W N T$ gene family, including WNT3A, WNT3, WNT5A, WNT7A, $W N T 8 A, W N T 9 B$, and $W N T 11$, have been thoroughly investigated. A previous study reported that among the 13 SNPs investigated in the WNT3A, WNT5A, WNT8A, WNT11, WNT3, and $W N T 9 B$ genes (in 463 Caucasian individuals), the rs142167 [P $=2.80 \mathrm{E}-05$; OR $=1.61$ (1.29$2.02)]$ and rs $9890413[\mathrm{P}=0.002 ; \mathrm{OR}=1.4(1.12-1.74)] \mathrm{SNPs}$ in $W N T 3$ were significantly associated with all clefts (Menezes et al., 2010). Chiquet et al. (2008) also discovered significant differences between the phenotypes of the rs9890413 SNP in the WNT3 gene in the European, American, and Hispanic population. These results indicate that the WNT3 gene is associated with NSCL/P.

In this study, the individuals carrying the T allele of rs3809857 showed (approximately) a 2-fold decreased risk of NSCL/P. FBAT analysis also showed under-transmission of the T allele and over-transmission of $\mathrm{G}$ allele from parents to their children. This indicated that the common $\mathrm{G}$ allele may be a risk factor, and the minor $\mathrm{T}$ allele may be a protective factor for NSCL/P. This is consistent with the results of the study conducted by Mostowska et al. (2012). The decreased risk for NSCL/P is inconsistent with the results of previous studies that suggested that variants of the WNT3 gene increase the risk of NSCL/P. Overall, our results 
indicated an association between the rs3809857 WNT3 variant and an unobserved causal variant; in addition, the former was determined to be non-functional.

The minor allele frequency (MAF) of rs9890413 in northeast China (MAF $=0.03$ ) is lower than in the European American (MAF $=0.363$ ), and Caucasian populations (MAF $=0.32$ ). This distinction may be attributed to the population heterogeneity between northeast China and the western countries. Despite a varying MAF value, a significant association was observed between the populations with respect to the rs9890413 SNP $[\mathrm{P}=0.002 ; \mathrm{OR}=2.369$ (1.354-4.145)], in the case-control analysis. Individuals carrying the $\mathrm{G}$ allele of the rs 9890413 SNP showed a 2.37-fold increased risk for NSCL/P. The FBAT analysis also showed overtransmission of the $\mathrm{G}$ allele in the rs9890413 SNP $(\mathrm{P}=0.00012)$. Our results supported the results of previous studies, suggesting that the WNT3 rs9890413 SNP is strongly associated with NSCL/P. On the contrary, Mostowska et al. (2012) genotyped fourteen polymorphisms in six $W N T$ genes in a population in Warsaw, and observed no correlations between rs 9890413 and NSCL/P $[\mathrm{P}=0.844 ; \mathrm{OR}=0.935(0.480-1.824)]$. This contradiction may be attributed to the genetic heterogeneity of different populations.

In the HBAT analysis, the G-G (order: rs3809857-rs9890413) haplotype was overtransmitted from the parents to the child. This result supported the theory that the WNT3 gene is associated with NSCL/P.

Our study has several limitations. Firstly, only two SNPs in the WNT3 gene were detected and investigated; the detection of more SNPs may strengthen our results. Secondly, the MAF of rs 9890413 was too low, and the number of cases and controls was relatively limited. Increasing the sample size would increase the robustness of our study. Thirdly, the gene-gene and geneenvironment interactions, which may explain the formation of NSCL/P, were not evaluated.

In this study, two SNPs in the WNT3 gene were tested in the northeast Chinese population. Our results continued to support the theory that the WNT3 genes may be associated with the occurrence of NSCL/P. This report details supplemental research of a previous study (Chiquet et al., 2008), and may be helpful in extending our understanding of the etiology of NSCL/P.

\section{Conflicts of interest}

The authors declare no conflict of interest.

\section{ACKNOWLEDGMENTS}

Research supported by the Scientific and Technological Project of Liaoning Province (Grant \#2009225007-7) and the Natural Science Foundation of Liaoning Province (Grant \#201102247). The authors would like to thank all the participants of this study (who donated blood samples), as well as the staff at the School of Stomatology, China Medical University (who assisted in the collection of blood samples).

\section{REFERENCES}

Beaty TH, Hetmanski JB, Fallin MD, Park JW, et al. (2006). Analysis of candidate genes on chromosome 2 in oral cleft case-parent trios from three populations. Hum. Genet. 120: 501-518.

Beaty TH, Murray JC, Marazita ML, Munger RG, et al. (2010). A genome-wide association study of cleft lip with and without cleft palate identifies risk variants near MAFB and ABCA4. Nat. Genet. 42: 525-529. 
Birnbaum S, Ludwig KU, Reutter H, Herms S, et al. (2009). Key susceptibility locus for nonsyndromic cleft lip with or without cleft palate on chromosome 8q24. Nat. Genet. 41: 473-477.

Brugmann SA, Goodnough LH, Gregorieff A, Leucht P, et al. (2007). Wnt signaling mediates regional specification in the vertebrate face. Development 134: 3283-3295.

Chiquet BT, Blanton SH, Burt A, Ma D, et al. (2008). Variation in WNT genes is associated with non-syndromic cleft lip with or without cleft palate. Hum. Mol. Genet. 17: 2212-2218.

Dixon MJ, Marazita ML, Beaty TH and Murray JC (2011). Cleft lip and palate: understanding genetic and environmental influences. Nat. Rev. Genet. 12: 167-178.

Feng C, Duan W, Zhang D, Zhang E, et al. (2014). A C392T polymorphism of the Wnt10a gene in nonsyndromic oral cleft in a northeastern Chinese population. Br. J. Oral Maxillofac. Surg. 52: 751-755.

Grant SF, Wang K, Zhang H, Glaberson W, et al. (2009). A genome-wide association study identifies a locus for nonsyndromic cleft lip with or without cleft palate on 8q24. J. Pediatr. 155: 909-913.

Lan Y, Ryan RC, Zhang Z, Bullard SA, et al. (2006). Expression of Wnt9b and activation of canonical Wnt signaling during midfacial morphogenesis in mice. Dev. Dyn. 235: 1448-1454.

Letra A, Menezes R, Granjeiro JM and Vieira AR (2009). AXIN2 and CDH1 polymorphisms, tooth agenesis, and oral clefts. Birth Defects Res. A Clin. Mol. Teratol. 85: 169-173.

Liu F and Millar SE (2010). Wnt/beta-catenin signaling in oral tissue development and disease. J. Dent. Res. 89: 318-330.

Lu Y, Liu Q, Xu W, Li Z, et al. (2013). TGFA and IRF6 contribute to the risk of nonsyndromic cleft lip with or without cleft palate in northeast China. PloS One 8: e70754.

MacDonald BT, Tamai K and He X (2009). Wnt/ $\beta$-catenin signaling: components, mechanisms, and diseases. Dev. Cell 17: 9-26.

Mangold E, Ludwig KU, Birnbaum S, Baluardo C, et al. (2010). Genome-wide association study identifies two susceptibility loci for nonsyndromic cleft lip with or without cleft palate. Nat. Genet. 42: 24-26.

Menezes R, Letra A, Kim AH, Kuchler EC, et al. (2010). Studies with Wnt genes and nonsyndromic cleft lip and palate. Birth Defects Res. A Clin. Mol. Teratol. 88: 995-1000.

Mossey PA and Modell B (2012). Epidemiology of oral clefts 2012: an international perspective. Front. Oral Biol. 16: 1-18.

Mossey PA, Little J, Munger RG, Dixon MJ, et al. (2009). Cleft lip and palate. Lancet 374: 1773-1785.

Mostowska A, Hozyasz KK, Biedziak B, Wojcicki P, et al. (2012). Genotype and haplotype analysis of WNT genes in non-syndromic cleft lip with or without cleft palate. Eur. J. Oral Sci. 120: 1-8.

Pan Y, Zhang W, Du Y, Tong N, et al. (2011). Different roles of two novel susceptibility loci for nonsyndromic orofacial clefts in a Chinese Han population. Am. J. Med. Genet. 155A: 2180-2185.

Song L, Li Y, Wang K, Wang YZ, et al. (2009). Lrp6-mediated canonical Wnt signaling is required for lip formation and fusion. Development 136: 3161-3171.

Yao T, Yang L, Li PQ, Wu H, et al. (2011). Association of Wnt3A gene variants with non-syndromic cleft lip with or without cleft palate in Chinese population. Arch. Oral Biol. 56: 73-78. 